\title{
Functional Outcome Analysis of Trochanteric Fractures Treated with Proximal Femoral Nail Antirotation II
}

\author{
G Vinoth Kumar ${ }^{1}$, R Amarnath², S Senthil Kumar ${ }^{3}$, B Thanigaiarasu ${ }^{4}$
}

\begin{abstract}
Background: The treatment of intertrochanteric fractures continues to be a challenge in orthopedic trauma, especially in geriatric population. Among the various generations of cephalomedullary nails, proximal femoral nail antirotation II (PFN A-II) is specifically designed for Asian population, with helical blade, modified proximal diameter, and modified mediolateral angle.

Aim: The aim of this study was to analyze the functional outcome of patients treated with PFN A-II using Harris hip score, at our institution. The study included 20 patients ( 11 males and 9 females).

Results: Among the 20 patients included in the study, outcome was excellent in 5, good in 11, fair in 4, and this is based on Harris hip score with the minimum follow-up period being 6 months. The average union time was 13 weeks, average Harris hip score was 82.3 . An abductor lurch was reported in one patient, postoperative thigh pain in two patients, infection in three patients, and bedsore in three patients.

Keywords: Asian population, Harris hip score, Helical blade, Intertrochanteric fracture, Proximal femoral nail, Proximal femoral nail antirotation II. Journal of Orthopedics and Joint Surgery (2019): 10.5005/jp-journals-10079-1003
\end{abstract}

\section{INTRODUCTION}

Intertrochanteric fracture is one of the most devastating injuries whose incidence increases with advancing age. ${ }^{1}$ These patients are more limited to home ambulation and become dependent for doing their basic and instrumental activities of daily living. Fifty percent of fractures around hip in elderly patients involves trochanteric fracture that are of unstable type. They are usually complicated with associated comorbidities such as osteoporosis, diabetes, hypertension, and renal failure. In such circumstances, nonoperative treatment is mainly reserved for poor medical candidates and nonambulant patients with minimal discomfort after fracture. Today operative treatment has largely replaced conservative measures and the goal is to achieve accurate or acceptable treatment. Anatomical and stable reduction was performed with rigid internal fixation in order to achieve early mobilization of patients and prevent complications of prolonged recumbence. Despite marked improvements in implant design, surgical technique, and patient care, intertrochanteric fractures continue to consume a substantial proportion of our healthcare resources and remain a challenge to date. ${ }^{2}$ Complications with intertrochanteric fractures arise primarily from fixation rather than union or delayed union because the intertrochanteric area is made of cancellous bones. ${ }^{3}$

The strength of the fracture fragment-implant assembly depends upon various factors including ${ }^{4}$ bone quality, fragment geometry, reduction, implant design, and implant placement. Among all these factors, surgeon can only modify the quality of the reduction, choice of implant, and its placement. A wide variety of treatment options are available for these fractures. The sliding hip screw device has been used for more than a decade for the treatment of these fractures, which may not be an ideal implant in all cases. ${ }^{5,6}$ Intramedullary load sharing device, namely, PFN, helps in early postoperative mobilization, weight-bearing, and ultimately the early fracture union. The proximal femoral nail antirotation II (PFN A-II) utilizes a helical blade instead of the conventionally used two screws. The helical blade is believed to provide stability, compression as well as rotational control of the fracture. Theoretically it compacts the bone during insertion

\begin{abstract}
${ }^{1-4}$ Department of Orthopaedic Surgery, Government Royapettah Hospital and Kilpauk Medical College, Chennai, Tamil Nadu, India

Corresponding Author: B Thanigaiarasu, Department of Orthopaedic Surgery, Government Royapettah Hospital and Kilpauk Medical College, Chennai, Tamil Nadu, India, Phone: +91 9894618266, e-mail: drarasuortho@gmail.com

How to cite this article: Kumar GV, Amarnath R, Kumar SS, et al. Functional Outcome Analysis of Trochanteric Fractures Treated with Proximal Femoral Nail Antirotation II. J Orth Joint Surg 2019;1(1): 22-26.

Source of support: Nil

Conflict of interest: None

into the neck and hence has higher cutout strength as compared to other devices. The differences are that the mediolateral angle is reduced from $6^{\circ}$ to $5^{\circ}$. Hence, there is less chance of implant failure especially in elderly, osteoporotic bones. Thus, PFN A-II is a modification of the conventional PFN, which reduces even the minimal complications associated with conventional PFN and also provides additional advantages.
\end{abstract}

\section{Materials and Methods}

A prospective study carried out at the Department of Orthopaedics, Government Royapettah Hospital and Kilpauk Medical College, Chennai, Tamil Nadu, India. Twenty patients were studied (21 hips-one patient had bilateral trochanteric fracture). The patients consisted of 11 males and 9 females whose age-group ranged from 45 to 85 years. Right femur was involved in 10 cases, left femur in nine patients, and bilateral in one patient. Mode of injury was road traffic accident in 10 patients and self-fall in 11 patients. Patients were classified based on Boyd and Griffin classification, fracture, with type II being predominant and type I being least. The classification was done based on the preoperative anteroposterior view with traction and internal rotation taken at the time of admission. The trauma to surgery interval was 3-7 days. 


\section{Inclusion Criteria}

- Patients above 18 years of age presenting to our casualty with intertrochanteric femoral fractures with all Boyd and Griffin types (I-IV)

- Both displaced and undisplaced fractures

- Fractures of less than 1 week duration

- Without any other associated fractures

\section{Exclusion Criteria}

- Fractures with nonunion changes

- Old malunited intertrochanteric fracture

- Patients with arthritic changes in hip joint

- Pathological fractures

All patients were operated on the fracture table under spinal anesthesia after the routine preoperative assessment.

\section{Postoperative Protocol}

- Routine postoperative protocol and chest physiotherapy.

- Hip and knee mobilization from the first postoperative day.

- Weight-bearing increased in a graded manner.

- Perioperative deep vein thrombosis (DVT) prophylaxis with enoxaparin.

- Suture removal on 12th postoperative day

- Regular follow-up with periodical X-rays at 3rd and 6th months.

Patients were evaluated clinically and radiologically at 3 weeks' interval for the first 3 months and thereafter monthly for the next 3 months and bimonthly for the next 12 months. During follow-up, the Harris hip score was evaluated at 3 and 6 months postoperatively, and the functional outcomes based on pain, function, absence of deformity, and range of motion were also assessed using Harris hip score. Collection of data was per the proforma with consent from patients.

\section{Results}

The time duration of surgery of the patients varied from 37 to 98 minutes. The number of fluoroscopy shots used during surgery was less than 10 shots in three patients, 10 to 15 shots in 13 patients, and more than 15 shots in five patients. The blood loss during surgery is also less in these patients, with less than $50 \mathrm{~mL}$ in one patient, between $50 \mathrm{~mL}$ and $100 \mathrm{~mL}$ in seven patients, between $100 \mathrm{~mL}$ and $150 \mathrm{~mL}$ in 12 patients, and more than $150 \mathrm{~mL}$ in one patient.

The Harris hip score grading was done and five patients were graded excellent, 11 as good, 4 as fair, and none as poor. The patient with bilateral trochanteric fracture was graded good. Among excellent, three patients were male and two female. Among good cases, seven were male and four were female, and among fair cases, one was male and three were female.

The type I fractures had excellent outcome; all type II had good outcome; and types III and IV had excellent, good, and fair outcomes. Radiographically the mean time for union was about 13.09 weeks. The time for union ranged from 12 to 16 weeks. The length of hospital stay varied from 5 to 24 days (with a mean of 11 days).

\section{Complications}

Bedsore occurred in three patients (one male and two female), superficial infection occurred in three male patients, which resolved with antibiotics, three patients had deep infection (two male and one female), which resolved with debridement and antibiotics, one female patient had abductor lurch, and in two patients (one male and one female) had postoperative hip pain. No cases of helical screw cutout, revision surgery, nonunion, or DVT were reported in our study.

\section{Summary of Results}

- Average operating time

62.6 minutes

- Average blood loss

$130 \mathrm{~mL}$

- Abductor lurch

- Postoperative hip pain

1 case

- Helical blade cutout

- Average fracture union

2

0

- Average image intensifier shots

13.0 weeks

13.5 shots

- Average Harries hip score at 6 months

\section{Case Illustrations}

See Figures 1 to 4 .

\section{Discussion}

The PFN A-II is an effectively designed intramedullary load-sharing device. It incorporates the principles and theoretical advantages of the Zicker nail, dynamic hip screw, and locked intramedullary nail, with modifications for Asian population.

Biomechanically PFN A-II, just like the conventional PFN, is more stiff and has shorter moment arm, i.e., from the tip of helical blade to the center of femoral canal, whereas the DHS has a longer moment arm but undergoes significant stress on weight-bearing and hence higher incidence of lag screw cutout and varus malunion. The larger proximal diameter (17 mm) of the PFN A-II compared with PFN (15 $\mathrm{mm}$ ) gives additional stiffness to the nail. Minimal blood loss, shorter operative time, early weight-bearing, less chances of implant failure, minimal fluoroscopy time, easier helical blade insertion (compared with cumbersome lag screw and derotation screw), less chances of postoperative hip pain, and better performance than any other implant in elderly osteoporotic patients are all the advantages of PFN A-II.

In the current study, the union rate was $100 \%$. No cases of preoperative and postoperative femoral fractures were reported. No cases of varus malunion were reported.

The average blood loss in patients treated with the PFN A-II nail was $130 \mathrm{~mL}$, ranging from 50 to $275 \mathrm{~mL}$. The results were comparable with Karapinar et al.'s study ${ }^{7}$ (Table 1).

Average operating time in our series was 62.6 minutes. In our initial cases, the operating time was on the higher range (range 43-82 minutes). With experience, the operating time reduced.

Table 1: Comparative study: Average blood loss

\begin{tabular}{ll}
\hline Study & Average blood loss $(\mathrm{mL})$ \\
\hline Karapinar et al. & \\
Zhang et al. $^{8}$ & 127 \\
Zhou and Chang $^{9}$ & 180 \\
Li et al. $^{10}$ & 180 \\
Our series & 131.86 \\
\hline
\end{tabular}



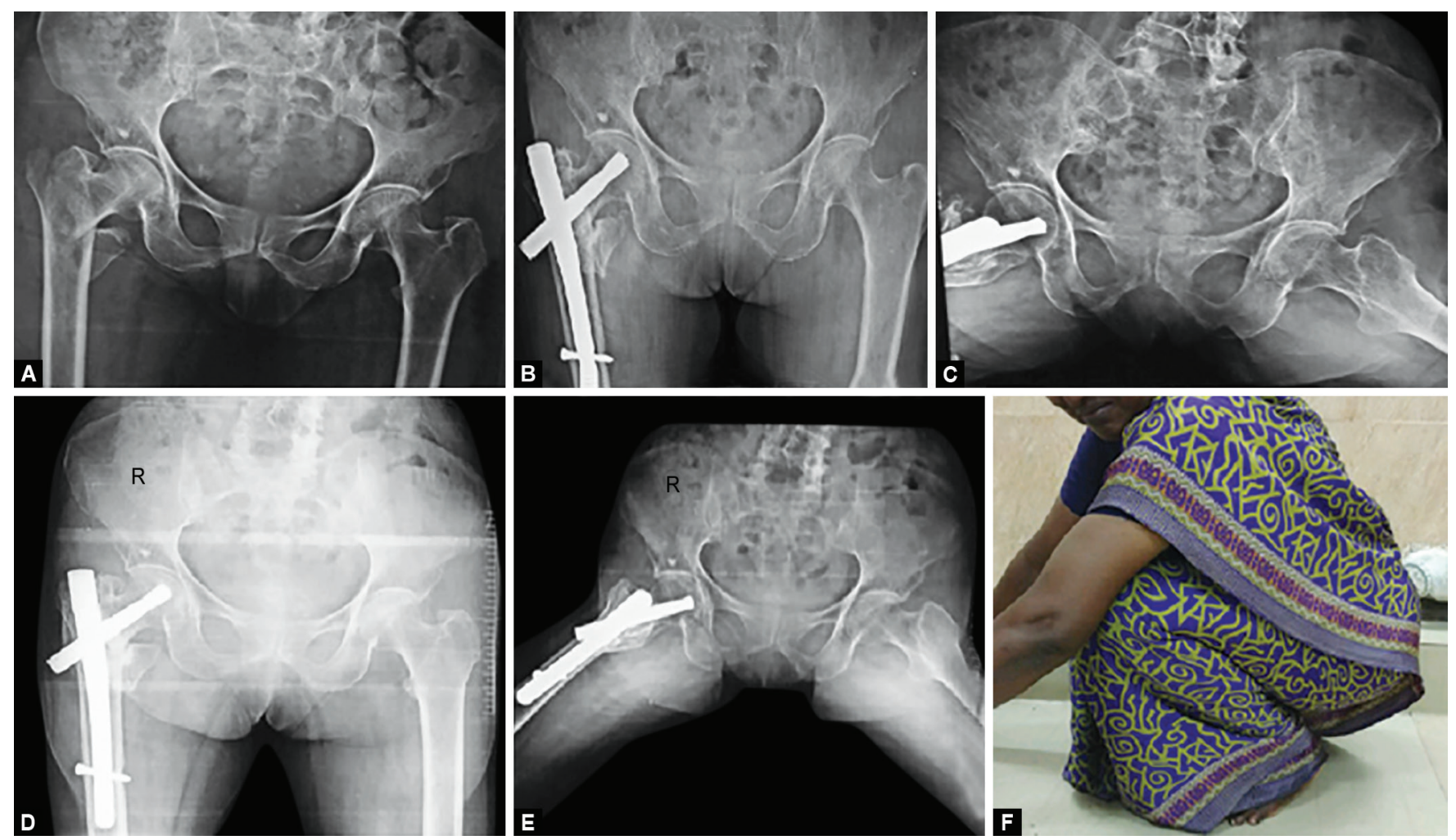

Figs 1 A to F: Case 1 illustrations
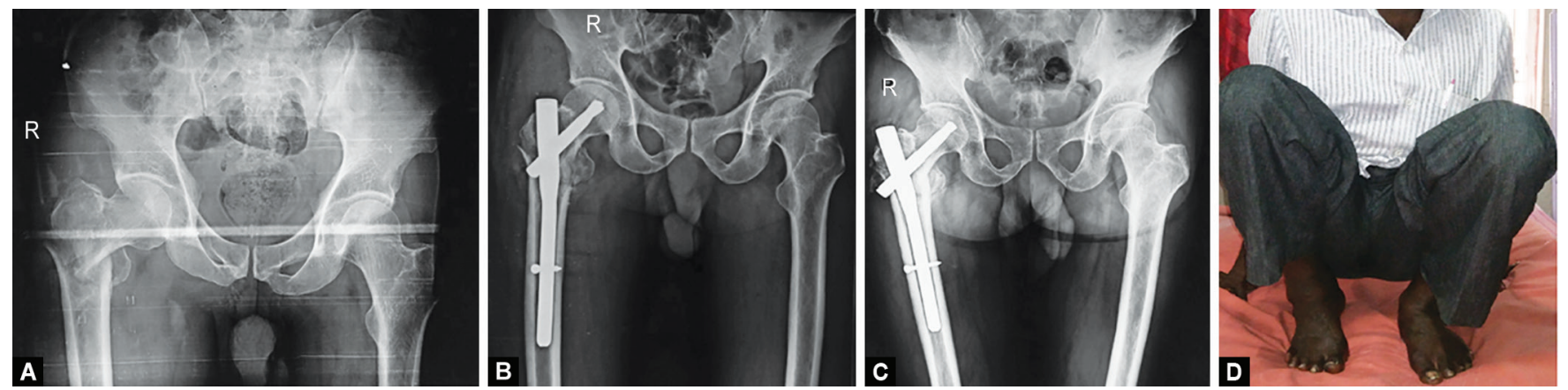

Figs 2A to D: Case 2 illustrations
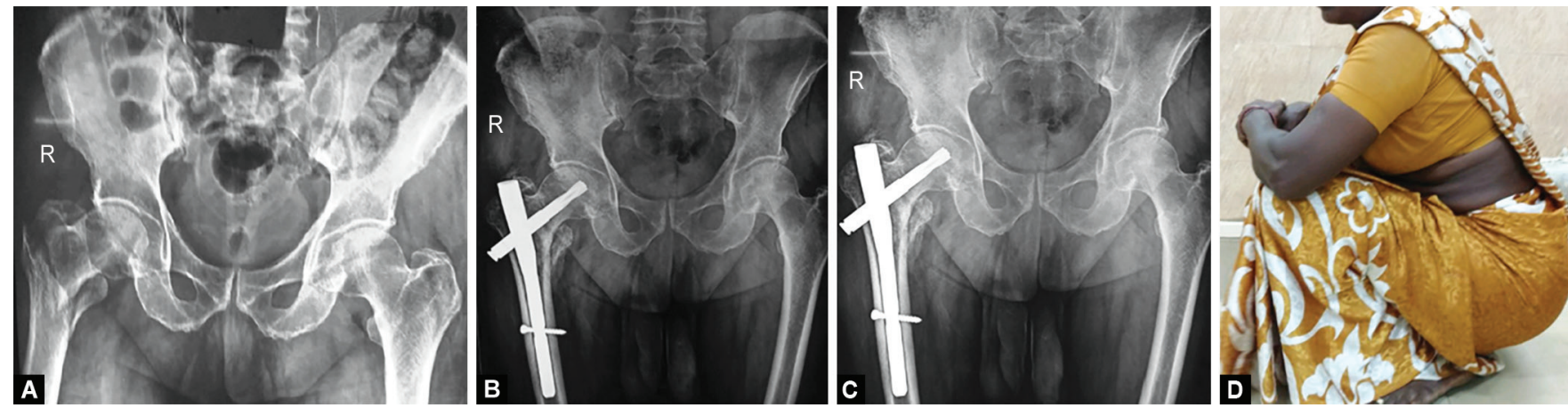

Figs $3 \mathrm{~A}$ to $\mathrm{D}$ : Case 3 illustrations

The operating time was more in types III and IV of Boyd and Griffin classification compared with other types.

Results were comparable to the series of Zhang et al. ${ }^{8}$ and Zhou et al. ${ }^{9}$ (Table 2).
The use of image intensifier was 13.5 shots in patients treated with PFN, which was comparable with the above-mentioned studies. In our study, it ranged from 8 to 20 shots. Fluoroscopy was more needed in types III and IV Boyd and Griffin classification. 

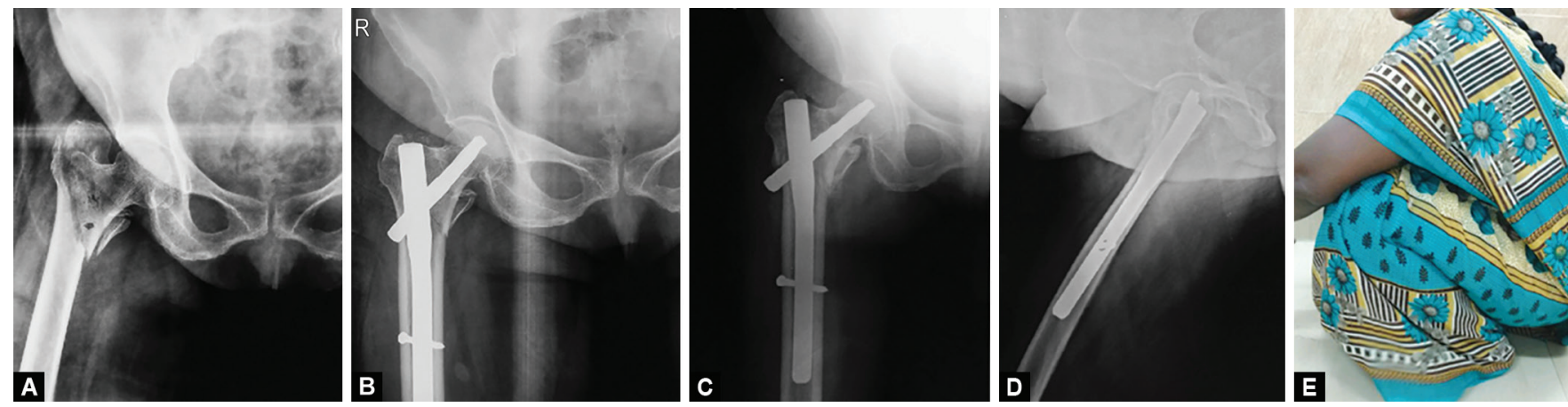

Figs $4 \mathrm{~A}$ to $\mathrm{E}$ : Case 4 illustrations

Table 2: Comparative study: Average operating time

\begin{tabular}{ll}
\hline Study & Average operating time (minutes) \\
\hline Karapinar et al. & 44.7 \\
Zhang et al. $^{8}$ & 55.6 \\
Zhou and Chang $^{9}$ & 68 \\
Li et al. $^{10}$ & 66.25 \\
Our series & 62.6 \\
\hline
\end{tabular}

Table 3: Comparative study: Average union time

\begin{tabular}{ll}
\hline Study & Average union time (weeks) \\
\hline Karapinar et al. ${ }^{7}$ & 14 \\
Zhang et al. $^{8}$ & 15.7 \\
Li et al. ${ }^{10}$ & 12.5 \\
Our series & 13.09 \\
\hline
\end{tabular}

Table 4: Comparative study: Average Harris hip score

\begin{tabular}{ll}
\hline Study & Average Harris hip score \\
\hline Karapinar et al. ${ }^{7}$ & 80.75 \\
Zhang et al. $^{8}$ & 81.90 \\
Li et al. ${ }^{10}$ & 86.19 \\
Our series & 82.3 \\
\hline
\end{tabular}

The time to union was 13.09 weeks in our study, ranging from 12 to 16 weeks. This was comparable to Karapinar et al. ${ }^{7}$ and $\mathrm{Li}$ et al. ${ }^{10}$ (Table 3).

The average Harris hip score in our patients was 79.8 (at the end of 3 months) and 82.3 (at the end of 6 months). Most of them were graded as "good" per Harris hip scoring. Fair scores were seen with higher age-group and higher Boyd and Griffin types.

The length of hospital stay in our study was 11 days (5-24 days), and it was comparable to Li et al. ${ }^{10}$ (10.8 days) and other studies.

In comparison, even other studies mentioned above have higher exposure fluoroscopy and greater blood loss in types III and IV of Boyd and Griffin (Table 4).

The complications in our study included bedsores, superficial and deep infections (which settled subsequently with intravenous antibiotics and debridement, respectively), abductor lurch, and postoperative hip pain. These were also present in other studies of PFN A-II.

A major complication of screw cutout was reported in few cases of other studies. Two cases of helical blade cutout (out of 42 patients) was reported by Karapinar et al. ${ }^{7}$ Our study did not report any complications of screw cutout or revision surgeries, because in our study all helical blades were placed per the tip apex distance as mentioned by Baumgaertner et al. ${ }^{11}$ Yet, our sample size is inadequate to report this complication.

No cases of non-union were reported in our study comparable to karapinar et al. ${ }^{7}$ wherein there was no reported cases of nonunion. Studies which reported non-union were highlighting that higher types (type III and IV) showed tendency toward nonunion.

Preoperative and postoperative femoral fractures have been documented in patients treated with PFN and PFN A-II. Multiple factors have been implicated such as implant design and operative technique. Decreases in implant curvature, diameter, over reaming of femoral canal by $1.5-2.0 \mathrm{~mm}$, insertion of implant by hand and meticulous placement of the distal locking screws without creating additional stress decrease the complication rate of femoral shaft fracture (Schipper et al.). ${ }^{12}$ Patients with narrow femoral canal and abnormal curvature of the proximal femur are relative contraindications to intramedullary implants (Halder et al.). ${ }^{2}$ Since these recommendations were followed in our series, we did not encounter any preoperative and postoperative femoral shaft fractures. A larger cohort of patients is necessary to document the incidence of preoperative and postoperative femoral shaft fractures, which is a limitation of our study.

In short, the PFN A-II is a better implant with specific design superior to conventional PFN and also with distinct advantages over other implants to treat intertrochanteric fractures. With adequate surgical technique, the advantages of the PFN A-II increase and the complication rate decreases.

\section{Conclusion}

Intramedullary nailing with the PFN A-II has distinct advantages over conventional PFN or DHS, such as shorter operating time and less blood loss for elderly, osteoporotic unstable trochanteric fractures.

Early mobilization and weight-bearing are allowed in patients treated with PFN A-II, thereby decreasing the incidence of bedsores, uremia, and hypostatic pneumonia.

The operative time is much lower compared with other procedures, which also contributes to less blood loss.

The incidence of postoperative femoral shaft fractures and nonunion rates in PFN A-II can be reduced by good preoperative planning and correct surgical technique, adequate reaming of the femoral canal, insertion of implant, and meticulous placement of distal locking screws.

The PFN A-II is a significant advancement in the treatment of trochanteric fractures, which has the unique advantage of closed reduction, preservation of fracture hematoma, minimal soft tissue damage during surgery, early rehabilitation, and early return to work. 


\section{References}

1. $\mathrm{Xu} Y Z$, Geng DC, Mao HQ, et al. A comparison of the proximalnail antirotation device and dynamic hip screw in the treatment of unstable pertrochanteric fracture. J Int Med Res 2010;38(4):1266-1275. DOI: $10.1177 / 147323001003800408$.

2. Halder SC. The Gamma nail for peritrochanteric fractures. J Bone Joint Surg Br 1992;74(3):340-344. DOI: 10.1302/0301-620X.74B3.1587873.

3. Akıncı $O$, Akalın $Y$, Reisoğlu $A$, et al. Comparison of long-term results of dynamic hip screw and $A O 130$ degrees blade plate in adult trochanteric region fractures. Acta Orthop Traumatol Turc 2010;44(6):443-451. DOI: 10.3944/AOTT.2010.2356.

4. Sadowski C, Lübbeke A, Saudan M, et al. Treatment of reverse oblique and transverse intertrochanteric fractures with use of an intramedullary nail or a 95 degrees screw-plate: a prospective, randomized study. J Bone Joint Surg Am 2002;84(3):372-381. DOI: 10.2106/00004623-200203000-00007.

5. Haidukewych G, Israel A, Berry D. Reverse obliquity fractures of the intertrochanteric region of the femur. J Bone Joint Surg Am 2001;83:643-650. DOI: 10.2106/00004623-200105000-00001. Kashid MR, et al. Int J Res Orthop 2016;2(4):354-363.

6. Cooper C, Campion G, Melton 3rd LJ. Hip fractures in the elderly: a world-wide projection. Osteoporos Int 1992;2(6):285-289. DOI: 10.1007/bf01623184.
7. Karapinar L, Kumbaraci M, Kaya A, et al. Proximal femoral nail antirotation (PFNA) to treat peritrochanteric fractures in elderly patients. Eur J Orthop Surg Traumatol 2012;22(3):237-243.

8. Zhang K, Zhang S, Yang J, et al. Proximal femoral nail vs dynamic hip screw in treatment of intertrochanteric fractures: a meta-analysis. Med Sci Monit 2014;20:1628-1633. DOI: 10.12659/MSM.890962.

9. Zhou J-Q, Chang S-M. Failure of PFNA: helical blade perforation and tip-apex distance. Injury 2012;43(7):1227-1228. DOI: 10.1016/j. injury.2011.10.024.

10. Li J, Cheng L, Jing J. The Asia proximal femoral nail antirotation versus the standard proximal femoral antirotation nail for unstable intertrochanteric fractures in elderly Chinese patients. Orthop Traumatol Surg Res 2015;101(2):143-146. DOI: 10.1016/j.otsr.2014. 12.011. Kyle RF, Gustilo RNB, Premer RF. Analysis of six hundred and twenty-two intertrochanteric hip fractures. J Bone Joint Surg Am 1979;61(2):216-221.

11. Baumgaertner MR, Curtin SL, Lindskog DM, et al. The value of the tip-apex distance in predicting failure of fixation of peritrochanteric fractures of the hip. J Bone Joint Surg Am 1995;77(7):1058-1064. DOI: 10.2106/00004623-199507000-00012.

12. Schipper IB, Steyerberg EW, Castelein RM, et al. Treatment of unstable trochanteric fractures: randomised comparison of the gamma nail and the proximal femoral nail. J Bone Joint Surg Br 2004;86(1):86-94. 